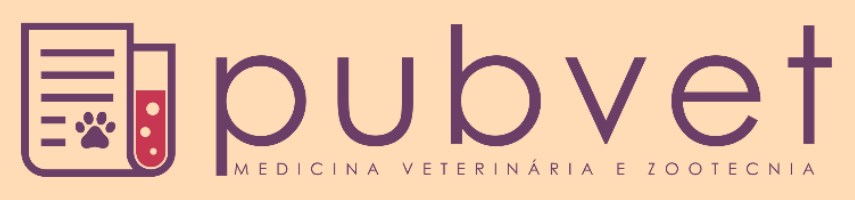

https://doi.org/10.31533/pubvet.v14n9a645.1-3

\title{
Deslocamento de flexura pélvica devido a lipoma intraluminal em cólon menor em equino: Relato de caso
}

\author{
Camila Rodrigues $\operatorname{Vargas}^{1 *}$, Adriéli $\operatorname{Matias}^{1}{ }^{1}$, Daniel Pereira dos $\operatorname{Santos}^{10}$, Julia Daniel \\ $\operatorname{Damiani}^{1}{ }^{\theta}$, Gabriel Pereira Berti ${ }^{2} \theta$, Guilherme Valente de Souza $^{3} \theta$, Henrique Mondardo \\ $\operatorname{Cardoso}^{49}$, Luciane Orbem Veronezi ${ }^{30}$ \\ ${ }^{I}$ Acadêmica de Medicina Veterinária, Centro Universitário Barriga Verde - UNIBAVE. Orleans - SC, Brasil. \\ ${ }^{2}$ Médico Veterinário do Hospital Veterinário Unibave, Centro Universitário Barriga Verde - UNIBAVE. Orleans - SC, Brasil. \\ ${ }^{3}$ Médico Veterinário, Docente, Centro Universitário Barriga Verde - UNIBAVE. Orleans - SC, Brasil. \\ ${ }^{4}$ Médico Veterinário, Mestrando Programa de Pós-Graduação em Medicina Animal-Equinos, Universidade Federal do Rio \\ Grande do Sul-RS, Brasil. \\ *Autor para correspondência, E-mail: camila.rvargas@hotmail.com
}

\begin{abstract}
Resumo. A Síndrome Cólica é um conjunto de sintomas e sinais clínicos que juntamente associados à histórico clínico do animal refletem aspectos fisiológicos no trato gastrointestinal que evidenciam o desconforto abdominal, resultando em enfermidades que afetam os equinos. O objetivo deste trabalho é relatar um caso de síndrome cólica causada por obstrução que, consequentemente, levou ao deslocamento de intestino grosso, nas porções de flexura pélvica e ceco. A paciente teve cólica recidiva, com causa primária de um lipoma encontrado no interior das alças intestinais, na região do cólon menor.
\end{abstract}

Palavras chave: gastrointestinal, laparotomia, lipoma, neoplasia

\section{Pelvic flexure dislocation due to intraluminal lower colon lipoma in a horse: Case report}

\begin{abstract}
Colic Syndrome is a set of clinical symptoms and signs that together with the animal's clinical history reflect physiological aspects in the gastrointestinal tract that evidence abdominal discomfort, resulting in diseases that affect horses. The objective of this paper is to report a case of colic syndrome caused by obstruction that, consequently, led to large bowel dislocation in the portions of the pelvic flexure and cecum. The patient had relapsed colic, with primary cause of a lipoma found inside the intestinal loops, in the lower colon region.
\end{abstract}

Keywords: gastrointestinal, laparotomy, neoplasia

\section{Luxación de la flexión pélvica debido a un lipoma intraluminal de colon inferior en equino: Reporte de caso}

Resumen. El Síndrome de cólico es un conjunto de síntomas y signos clínicos que, junto con la historia clínica del animal, reflejan aspectos fisiológicos en el tracto gastrointestinal que evidencian molestias abdominales, lo que resulta en enfermedades que afectan a los caballos. El objetivo de este trabajo es informar un caso de síndrome de cólico causado por una obstrucción que, en consecuencia, condujo a una luxación del intestino grueso en las porciones de la flexión pélvica y el ciego. El paciente tenía cólico recurrente, con causa primaria de un lipoma que se encuentra dentro de las asas intestinales en la región inferior del colon.

Palabras clave: gastrointestinal, laparotomía, lipoma, neoplasia 


\section{Introdução}

Uma das alterações do trato gastrointestinal dos equinos de grande ocorrência na clínica médica e cirúrgica é a síndrome cólica, caracterizada pela manifestação de intensa dor abdominal. No entanto, essa dor é um sinal inespecífico, e sua origem está relacionada a alterações patológicas do sistema gastrointestinal ou, até mesmo a outras alterações que não envolvam este sistema. Estas, geralmente são acompanhadas de afecções sistêmicas capazes de acarretarem a morte do animal caso não haja intervenção clínica ou até mesmo cirurgia (Jesus, 2018).

Comumente, na clínica médica equina, podemos acompanhar casos de síndrome cólica por obstrução e deslocamento de cólon maior - porção do intestino grosso - que possui predisposição em porções mais estreitas, como flexura pélvica (Oliveira et al., 2014) e essa obstrução pode-se dar por vários fatores, inclusive a presença de neoplasmas como lipomas (Freeman \& Schaeffer, 2001). Os cavalos acometidos por esta patologia apresentam sintomas típicos de cólica por lesão obstrutiva e o diagnóstico se dá na laparotomia exploratória, quando, geralmente, o lipoma e a parte do intestino envolvido são removidos (Blikslager et al., 1992; Edwards \& Proudman, 1994).

Os lipomas são neoplasias benignas formadas por adipócitos (células de tecido adiposo) que podem surgir em qualquer região do corpo. Os lipomas dos animais domésticos surgem preferencialmente no tecido subcutâneo (na região do peito, abdômen, esterno, axilas e pernas), mas podem ser vistos na cavidade abdominal principalmente em equinos e suínos (Blikslager et al., 1992; Edwards \& Proudman, 1994).

\section{Material e métodos}

Paciente da espécie equina, fêmea, da raça Mangalarga Machador, 2,5 anos de idade e pesando aproximadamente $370 \mathrm{~kg}$ foi atendido no hospital veterinário em abril de 2018 tendo como queixa principal episódio de dor e desconforto abdominal. No exame clínico a frequência cardíaca 60 batimentos por minuto (BPM), frequência respiratória 20 respirações por minuto (RPM), mucosas normocoradas, tempo de preenchimento capilar 2 segundos, temperatura corporal $38,1^{\circ} \mathrm{C}$. Na auscultação intertinal paciente apresentou hipomotilidade e ausência de descargas. Na sondagem nasogástrica não houve presença de refluxo. Por meio da palpação retal evidenciou-se deslocamento da flexura pélvica. Foi colhido sangue para realização de hemograma completo, onde todos os resultados estavam normais. O paciente recebeu fluidoterapia e dipirona $(20 \mathrm{mg} / \mathrm{kg}$, IV), óleo mineral, sulfato de magnésio e bisacodil via sonda.

$\mathrm{Na}$ inspeção abdominal a flexura pélvica estava deslocada sendo realizado o reposicionamento das alças intestinais, sem a necessidade de enterotomia. No pós-operatório a paciente recebeu fluidoterapia com ringer lactato, cloridrato de Ceftiofur (4,0 mg/kg, SID, intramuscular (IM), 60 por 7 dias), flunixin meglumine (1,1mg/kg, BID, IM, 4 dias) e previcox, (113 mg, VO, 10 dias) e, suplementação vitamínica (Floramax $^{\circledR}$, IM, por 7 dias). A limpeza da ferida era realizada uma vez ao dia e a retirada dos pontos ocorreu a partir do $15^{\circ}$ dia do pós-cirúrgico e de forma intercalada. $\mathrm{O}$ animal recebeu alta após a retirada de todos os pontos.

Em maio de 2019 o paciente retornou ao hospital veterinário apresentando os mesmos sinais clínicos do primeiro atendimento. No exame clínico a frequência cardíaca $62 \mathrm{bpm}$, frequência respiratória 24 $\mathrm{rpm}$, mucosas normocoradas, tempo de preenchimento capilar 2 segundos, temperatura corporal $38,0^{\circ} \mathrm{C}$. $\mathrm{Na}$ auscultação intestinal paciente apresentou hipomotilidade. Na palpação retal suspeitou-se de compactação, deslocamento de flexura pélvica e ceco. Realizou-se coleta sanguínea para exames laboratoriais, onde, os mesmos também apresentaram valores dentro do normal, fluidoterapia com ringer lactato, administração de flunixin meglumine $(1,1 \mathrm{mg} / \mathrm{kg}, \mathrm{IV}) . \mathrm{O}$ animal foi novamente submetido a laparotomia exploratória.

Durante a exploração abdominal constatou-se retroflexão de flexura pélvica, que foi exteriorizada juntamente com os cólons dorsal esquerdo e ventral direito onde foi realizado enterotomia na região da flexura pélvica para desfazer a compactação. $\mathrm{O}$ cólon menor também apresentava compactação e submetido a enterotomia. As enterorrafias foram realizadas com dois padrões de suturas e fio absorvível mono filamentar. Durante a inspeção do abdômen evidenciou-se a presença de uma massa de aproximadamente $3,5 \mathrm{~cm}$ de diâmetro e $8,5 \mathrm{~cm}$ de comprimento no lúmen do colón menor. Por se tratar de uma região de difícil acesso cirúrgico com riscos consideráveis de contaminação da cavidade 
abdominal, optou-se por realizar punção aspirativa por agulha fina (PAAF), para exame citológico e, constatação da origem celular do nódulo.

No pós-operatório o animal permaneceu na fluidoterapia com ringer lactato, sendo administrado gentamicina $(6,6 \mathrm{mg} / \mathrm{kg}, \mathrm{IM}, \mathrm{BID}, 5$ dias$)$, flunixin meglumine $(1,1 \mathrm{mg} / \mathrm{kg}, \mathrm{BID}, \mathrm{IM}, 3 \mathrm{dias})$, metronidazol, (20 mg/kg, VO, BID, 10 dias), omeprazol, (VO, SID, 30 dias), metoclopramida $(0,25$ $\mathrm{mg} / \mathrm{kg}$, IM, dose única) e suplementação vitamínica (Bionew ${ }^{\circledR}$, dose única, VO e Laviz pró+pré ${ }^{\circledR}, 4$ dias, IM). A limpeza da ferida era realizada uma vez ao dia e a retirada dos pontos se deu a partir do $15^{\circ}$ dia após o procedimento cirúrgico e de forma intercalada. O animal teve alta após 28 dias de internação.

\section{Resultados e discussão}

Segundo Thomassian (2005), o procedimento cirúrgico é indicado quando não houver diagnóstico clínico definitivo ou que haja suspeita de alguma alteração no aparelho digestivo. Nesse caso, constatado o deslocamento e compactação no exame clínico através da palpação retal realizou-se o procedimento cirúrgico no qual verificou-se a presença do neoplasma.

$\mathrm{Na}$ avaliação citológica do aumento de volume intestinal intraluminal observou-se uma quantidade moderada de restos de debris celulares, associados a gordura livre, lipócitos maturos e possíveis lipoblastos, apresentando aspecto gorduroso. Os lipócitos possuem núcleos picnóticos que são comprimidos contra a região da membrana celular pela presença de enormes gotículas de gordura. Como resultado desta condição os esfregaços possuem uma aparência oleosa e não secam o que fícou bem evidenciado neste caso. As alterações cito patológicas caracterizaram um tumor mesenquimal de lipócitos (Lipoma).

Geralmente, o lipoma e a parte do intestino acometido são retirados no procedimento cirúrgico, porém, como o cólon menor é uma região difícil de ser exposta, sua remoção é muito difícil e, associado ao risco de contaminação de toda a cavidade abdominal, optou-se por não o remover.

\section{Considerações finais}

As cólicas intestinais em equinos é uma emergência clínica de grande ocorrência na rotina veterinária e pode ser oriunda de diversas causas, dentre elas pode-se destacar a presença de tumores intraluminais. Nos casos de equinos com lipoma nos órgãos da cavidade abdominal estes, geralmente são encontrados em laparotomia exploratória, e são removidos juntamente com parte do intestino envolvida, ou ainda, dependendo do caso estes são achados durante a necropsia (Edwards \& Proudman, 1994).

\section{Referências bibliográficas}

Blikslager, A. T., Bowman, K. F., Haven, M. L., Tate Junior, L. P., \& Bristol, D. G. (1992). Pedunculated lipomas as a cause of intestinal obstruction in horses: 17 cases (1983-1990). Journal of the American Veterinary Medical Association, 201(8), 1249-1252.

Edwards, G. B., \& Proudman, C. J. (1994). An analysis of 75 cases of intestinal obstruction caused by pedunculated lipomas. Equine Veterinary Journal, 26(1), 18-21. https://doi.org/10.1111/j.20423306.1994.tb04324.x.

Freeman, D. E., \& Schaeffer, D. J. (2001). Age distributions of horses with strangulation of the small intestine by a lipoma or in the epiploic foramen: 46 cases (1994-2000). Journal of the American Veterinary Medical Association, 219(1), 87-89. https://doi.org/10.2460/javma.2001.219.87.

Jesus, C. N. R. (2018). Estudo retrospectivo dos Casos de Cólica do Hospital Veterinário Luís Leigue do período de junho de 2015 a setembro de 2018. Curitibanos, SC.

Oliveira, C. M., Ribeiro, I. B., Gadelha, I. C. N., Calado, E. B., Paula, V. V., Barrêto-Junior, R. A., Dias, R. V. C., \& Câmara, A. C. L. (2014). Achados clínicos-epidemiológicos de 25 casos decólica em equídeos no Rio Grande do Norte. Acta Veterinaria Brasilica, 8(4), 290-294.

Thomassian, A. (2005). Enfermidades dos cavalos. Livraria Varela.

Recebido: 6 de março, 2020.

Aprovado: 3 de abril, 2020.

Disponível online: 17 de agosto, 2020.
Licenciamento: Este artigo é publicado na modalidade Acesso Aberto sob a licença Creative Commons Atribuição 4.0 (CC-BY 4.0), a qual permite uso irrestrito, distribuição, reprodução em qualquer meio, desde que o autor e a fonte sejam devidamente creditados. 